\title{
Phase Separation in Lipid Membranes
}

\author{
Frederick A. Heberle and Gerald W. Feigenson \\ Department of Molecular Biology and Genetics, Field of Biophysics, Cornell University, \\ Ithaca, New York 14853 \\ Correspondence: gwf3@cornell.edu
}

Cell membranes show complex behavior, in part because of the large number of different components that interact with each other in different ways. One aspect of this complex behavior is lateral organization of components on a range of spatial scales. We found that lipidonly mixtures can model the range of size scales, from approximately $2 \mathrm{~nm}$ up to microns. Furthermore, the size of compositional heterogeneities can be controlled entirely by lipid composition for mixtures such as 1,2-distearoyl-sn-glycero-3-phosphocholine (DSPC)/1,2dioleoyl-sn-glycero-3-phosphocholine (DOPC)/1-palmitoyl-2-oleoyl-sn-glycero-3-phosphocholine (POPC)/cholesterol or sphingomyelin (SM)/DOPC/POPC/cholesterol. In one region of special interest, because of its connection to cell membrane rafts, nanometer-scale domains of liquid-disordered phase and liquid-ordered phase coexist over a wide range of compositions.

\section{SCALES OF SPATIAL ORGANIZATION OF CELL MEMBRANES}

Cell membranes have daunting complexity, including but not limited to the scales of spatial organization that emerge from a complex system. Complex behavior arises in part from the large number of different kinds of lipids and proteins, the different ways in which these membrane components interact with each other and with the rest of the cell, and the dynamic processes that locally and dramatically change significant fractions of the membrane. To appreciate the nature of this behavior, various key aspects of the complexity must be examined. Although a complete picture has not yet emerged, the available data are compelling that compositional heterogeneity, rather than random mixing of membrane lipids and proteins, describes cell membranes (Lingwood and Simons 2010). Here, we focus on one specific subset of complex membrane behavior: lateral heterogeneity based on lipid-lipid interactions in multicomponent bilayer mixtures. The impetus for examining such mixtures is the apparent connection between the coexistence of liquiddisordered (Ld) and liquid-ordered (Lo) phases observed in some simple, lipid-only bilayers, and the properties of lipid rafts observed in some cellular membranes. One possible starting point for experimental study is to find the simplest system that shows a wide range of organization comparable to what is found in cells, that is, from nanometers to microns. This turns out

Editor: Kai Simons

Additional Perspectives on The Biology of Lipids available at www.cshperspectives.org

Copyright (C) 2011 Cold Spring Harbor Laboratory Press; all rights reserved; doi: 10.1101/cshperspect.a004630

Cite this article as Cold Spring Harb Perspect Biol 2011;3:a004630 
to be a four-component lipid bilayer mixture at equilibrium, containing cholesterol.

Membranes of living cells show spatial organization on all relevant size scales, ranging from molecular lengths to the dimensions of the cell itself. The plasma membrane is the site of controlled crossing of molecules and ions between the cell and the environment, and is the anisotropic medium in which proteins find their partners and catalyze chemical reactions. The size scale for such molecular interactions is in the range of a few nanometers. Some larger bilayer environments are known to have specialized purposes, for example, membrane regions on the order of $50-100 \mathrm{~nm}$ that characterize caveolae and other sites of membrane fusion and endocytosis. A larger, micron-size scale describes the clustering of proteins and lipids in the membrane site of an immune synapse (Manz and Groves 2010). At a scale of tens of microns, epithelial cell plasma membranes are organized into the very different morphological and compositional regions of apical and basolateral domains (Simons and van Meer 1988).

Small size scales of organization in a cell membrane are difficult to characterize experimentally. Several techniques have proven useful for detecting heterogeneities on the scale of such small collections of molecules, although not providing quantitative size measurement. Methods that are sensitive to the immediate environment of a molecule are probing a very short spatial scale, $\sim 1 \mathrm{~nm}$. Fluorescence techniques including anisotropy (Kinosita et al. 1984), lifetime (Haluska et al. 2008), quantum yield (Zhao et al. 2007a), and quenching by spin-labels (London and Feigenson 1981), as well as the ESR spectrum itself (Schneider and Freed 1989), are such highly locally sensitive methods. Although first-order phase separation creates different environments on both small and large spatial scales, simple nonideal lipid mixing also creates different local environments at the $\sim 1 \mathrm{~nm}$ scale. Experimental sensitivity to such small heterogeneities is needed, along with quantitative measure of spatial scale and (if possible) lifetime of any such domains. Superresolution imaging is highly promising in this regard. In a recent study, STED microscopy of fluorescently labeled lipids revealed transient trapping of sphingomyelin for an average time of $\sim 15 \mathrm{msec}$ within regions of diameter $<20$ $\mathrm{nm}$ (Sahl et al. 2010). Of course, these STEDdetected domains themselves must last longer than $15 \mathrm{msec}$. This highly significant finding rules out simple nonideal mixing, which would result in molecular clusters that dissipate on a timescale of tens of microseconds (Abney and Scalettar 1995). In addition to techniques employing a single probe species, FRET between donor and acceptor fluorophores is in principle capable of providing a measure of domain size in the range $1-10 \times \mathrm{R}_{0}$, which is in the range of 2-80 $\mathrm{nm}$ for available membrane fluorescent probes (Feigenson and Buboltz 2001). Finally, we note that any technique involving addition of an extrinsic probe carries the caveat that the probe molecule might perturb the native spatial organization of the membrane.

\section{MODELING SPATIAL ORGANIZATION IN CELL MEMBRANES}

The size scales, time scales, and patterns of heterogeneity found in membranes of live cells can be treated in terms of general physical principles (Seul and Andelman 1995; Imperio and Reatto 2006; Elson et al. 2010; Fan and Sammalkorpi 2010). One way to organize such general considerations is to classify the driving forces as equilibrium or nonequilibrium processes. For separated phases at equilibrium, competing interactions can give rise to patterns of spatial organization with variations in size, lifetime, and morphology. For example, when line tension dominates any other interactions that depend on domain (perimeter) size, coexisting liquid phases round up into large circular domains containing vast numbers of molecules to minimize their perimeter. This simple morphology can be modulated when interactions of sufficient magnitude compete with line tension, so that patterns appear-a maze of stripes, tiny round domains, curved lines, and branched lines (Seul and Andelman 1995). Also at equilibrium, and depending on proximity to a critical point, fluctuating domains showing a range of size and timescales appear (Honerkamp-Smith et al. 2008). 
Processes occurring far from equilibrium can strongly influence the nature of coexisting domains. An especially simple case is the failure of phase-separated Ld + Lo macroscopic domains to merge into an equilibrium large domain because of a high-energy barrier from membrane curvature at the domain boundaries (Semrau et al. 2009). Nanodomains can also be stabilized by entropy: If line tension is sufficiently low, the entropic penalty for merging many small domains into a single large domain can produce a "quasi-equilibrium" state of domains smaller than $50 \mathrm{~nm}$ (Frolov et al. 2006). A more complex and interesting process, also far from equilibrium and connected to other events in the cell, is the removal and delivery of patches of membrane having a composition distinct from that of the larger membrane. This dynamic process can generate a wide range of domain sizes and morphologies (Fan and Sammalkorpi 2010).

\section{LIPIDS FOUND IN CELL MEMBRANES}

Considering only the lipids (a mixture chosen by evolution in part to spontaneously form the bilayer phases that are the fundamental cell membrane structure), a first step for study of heterogeneity is to find the lipid composition of cell membranes (van Meer et al. 2008). A complete analysis would involve assaying the lipids of the given cell membrane, both qualitatively and quantitatively, and finding the particular composition of each leaflet. The latter has been a vexing problem for decades. Whole membrane assays are more feasible, and largescale efforts from "lipidomics" initiatives (Wenk 2005) have been successful in purifying particular cell membranes and measuring their overall composition, albeit without being able to characterize separate leaflet compositions so far. Different types of lipids have been found to predominate in different organisms, and in different membranes within a given cell. Even with so much new information, the connection between events of cell biology and particular lipid compositions - a kind of "lipid role assignment" — can be made in but a few cases. These exceptions include the phosphoinositide binding of particular proteins to the plasma membrane inner leaflet (McLaughlin and Murray 2005) and polyunsaturated acyl chains of lipids in the retinal rod, which are needed for full activity of rhodopsin (Polozova and Litman 2000). More information is known about plasma membranes than about any other cell membranes, making the plasma membrane a starting point for thinking about lipid roles in mammalian cell membranes.

In the favorable case that a particular membrane can be obtained in high purity, the overall lipid composition can be found. In comparison with the total lipids of a cell, two categories of lipids stand out for their abundance in the plasma membrane compared with other cell membranes: high melting temperature lipids, in particular sphingomyelin and gangliosides, and cholesterol (van Meer et al. 2008). These types of lipids are always present in the plasma membrane along with much lower melting temperature lipids, which in the outer leaflet are mostly phosphatidylcholines (Gennis 1989). After so many years of effort, it is perhaps surprising that the precise lipid composition has not been separately determined for outer and inner leaflets for any plasma membrane. Because the best understood case is that of the mature human erythrocyte, we will take these results as an approximate model for other mammalian plasma membranes: the outer leaflet has all of the gangliosides, the great majority of the sphingomyelin (SM), and much of the phosphatidylcholine (PC) and cholesterol; the inner leaflet has most of the membrane's phosphatidylethanolamine (PE), possibly all of its phosphatidylserine (PS) and phosphatidylinositol (PI) and some PC, and much cholesterol (Gennis 1989). It is important to remember that these numbers reflect an average over the entire membrane. Even if we know the composition of a leaflet, this does not imply that exactly this composition is found at all locations over the entire membrane surface, (e.g., apical and basolateral regions of an epithelial cell). In addition, many other lipid components, including the plasmalogen versions of glycerophospholipids, are also present. The phase behavior of these molecules in mixtures with other membrane components is largely unexplored. 
A more complete story of plasma membrane behavior should describe both the separate behavior of each leaflet, and the coupling between the leaflets (Collins and Keller 2008; Kiessling et al. 2009). The understanding of this "asymmetry" of the bilayer membrane is still at an early stage. Almost the entirety of what we know about the phase behavior of the lipid mixture in each leaflet of the plasma membrane comes from study of mixtures that separately model each leaflet.

\section{CAN NEGLECTING MEMBRANE PROTEINS BE A STARTING POINT FOR SIMPLE MODELS?}

Proteins and lipids comprise approximately equal fractions of the membrane, an obvious problem for the relevance of lipid-only mixtures to model a cell membrane. Yet, a closer inspection of the protein mass distribution reveals that the majority (perhaps $80 \%-85 \%$ ) is located within the aqueous milieu either outside or inside the cell. The remaining $\sim 15 \%-20 \%$ of the protein mass is located within the lipid bilayer (Sheetz 1993). This consideration leads to an estimate that the membrane bilayer, which we can define as the matter bounded by the lipid headgroups of each leaflet, is $10 \%-15 \%$ protein. Indeed, the very nature of the membrane as a bilayer phase is entirely a property of the lipids, not the proteins. Therefore, lipid-only models are likely to inform about some aspects of the nature of cell membranes.

\section{INFORMATION IN LIPID COMPOSITIONAL PHASE DIAGRAMS}

The history of composition-dependent phase diagrams of lipid bilayer mixtures dates from the 1960s (Phillips et al. 1970). Early experiments showed that single-component bilayers exist in a gel $(\mathrm{L} \beta)$ phase at low temperatures, characterized by a high trans/gauche conformer ratio in the hydrocarbon chains, as well as long-range twodimensional positional order. Following heating, these bilayers will ultimately pass through a firstorder melting transition to a disordered fluid phase (Ld), accompanied by a decrease in chain segmental order and the loss of long-range correlations in lipid position. Subsequent work revealed transitions to a third lamellar phase (the liquid-ordered, or Lo phase), found only in the presence of substantial amounts of cholesterol, and possessing extraordinary properties: the absence of long-range positional order as in a fluid, but with a high degree of chain segmental order as in a gel (Wu and McConnell 1975; Vist and Davis 1990). Early work also revealed that reliable data could be obtained to establish simple correlations, for example, that more structurally dissimilar lipids in a mixture give rise to wider regions of immiscibility (Tenchov and Koynova 1985; Huang and Li 1999).

The apparent connection between the membrane raft hypothesis and coexistence of distinct fluid phases ( $\mathrm{Ld}+\mathrm{Lo})$ in model systems spurred the study of biomimetic bilayer mixtures in the previous decade, with the goal of solving complete phase diagrams. The minimal systems that maintain biological relevance yet are amenable to relatively complete study contain both high-melting and lowmelting temperature $\left(T_{M}\right)$ lipids and cholesterol (Veatch and Keller 2003). A rich phase behavior is found in these systems, including a large region of coexisting ordered- and disordered-liquid phases at biologically relevant cholesterol concentrations (Marsh 2009). Furthermore, the size distribution of phase domains in the $\mathrm{Ld}+$ Lo region depends on the low- $T_{M}$ lipid: Strongly perturbing lipids like DOPC or diphytanoyl-PC generate micron-sized domains (Veatch et al. 2006; Zhao et al. 2007a), whereas biologically abundant low- $T_{M}$ lipids, such as POPC or 1-stearoyl-2olyeoyl-sn-glycero-3-phosphocholine (SOPC), yield nanometer-sized domains (de Almeida et al. 2003; Pokorny et al. 2006). In the following discussion, we consider the interactions that give rise to these phenomena.

\section{Lipid Clustering from Nonideal Mixing in Single-Phase Regions}

We begin by addressing cell membrane organization in the small size scale range of 5$200 \mathrm{~nm}$. A $200 \mathrm{~nm}$ bilayer patch contains about 
Phase Separation in Lipid Membranes

60,000 lipids per leaflet. A domain of this size is certainly large enough to be considered a phase, or even to have coexisting phases within the domain. But what is the nature of a $5 \mathrm{~nm}$ patch containing only approximately 40 molecules?

The thermodynamics of mixing, together with Monte Carlo computer simulations, provides a powerful, systematic basis for examination of lipid distributions based on lipid-lipid interaction energies. A simple visual inspection of Monte Carlo snapshots at equilibrium, such as those shown in Figures 1 and 2, is instructive. Cell membranes are far from random mixtures of lipids and proteins, but we start by considering exactly this case: an ideal mixture of, for example, phosphatidylcholines, defined by an excess mixing energy $\Delta E m=0$ (Guggenheim 1952). Figure 1A shows that, for an ideal equimolar binary mixture with only nearestneighbor interactions (e.g., no long-range or higher order interactions), groupings of three to five like lipids in each leaflet are commonly observed (Huang and Feigenson 1993). Firstorder phase separation occurs when $\Delta E m$ exceeds $0.55 \mathrm{kT}$ (Guggenheim 1952), and results in large phase domains seen in Figure 1D,E. Intermediate values of $\Delta E m$ generate small clusters. For example, with $\Delta E m=0.4 \mathrm{kT}$, the sizes of the numerous clusters are in the range 10 to 20 lipids (see Fig. 1B), a "domain size" of $\sim 3 \mathrm{~nm}$ (Huang and Feigenson 1993). At $\Delta E m$ $=0.5 \mathrm{kT}$, numerous clusters of approximately 100 lipids form, as shown in Figure 1C,F. Such small clusters from nonideal mixing have a short lifetime on the order of microseconds, disappearing and forming again elsewhere after many nearest-neighbor exchanges, each $\sim 10^{-7}$ s (Abney and Scalettar 1995).

Further insight into lipid mixing comes from considering the more complex behavior of multicomponent bilayer mixtures. Figure 2 shows snapshots of mixing behavior in which the interaction energies are held constant, but the concentration of a third component is

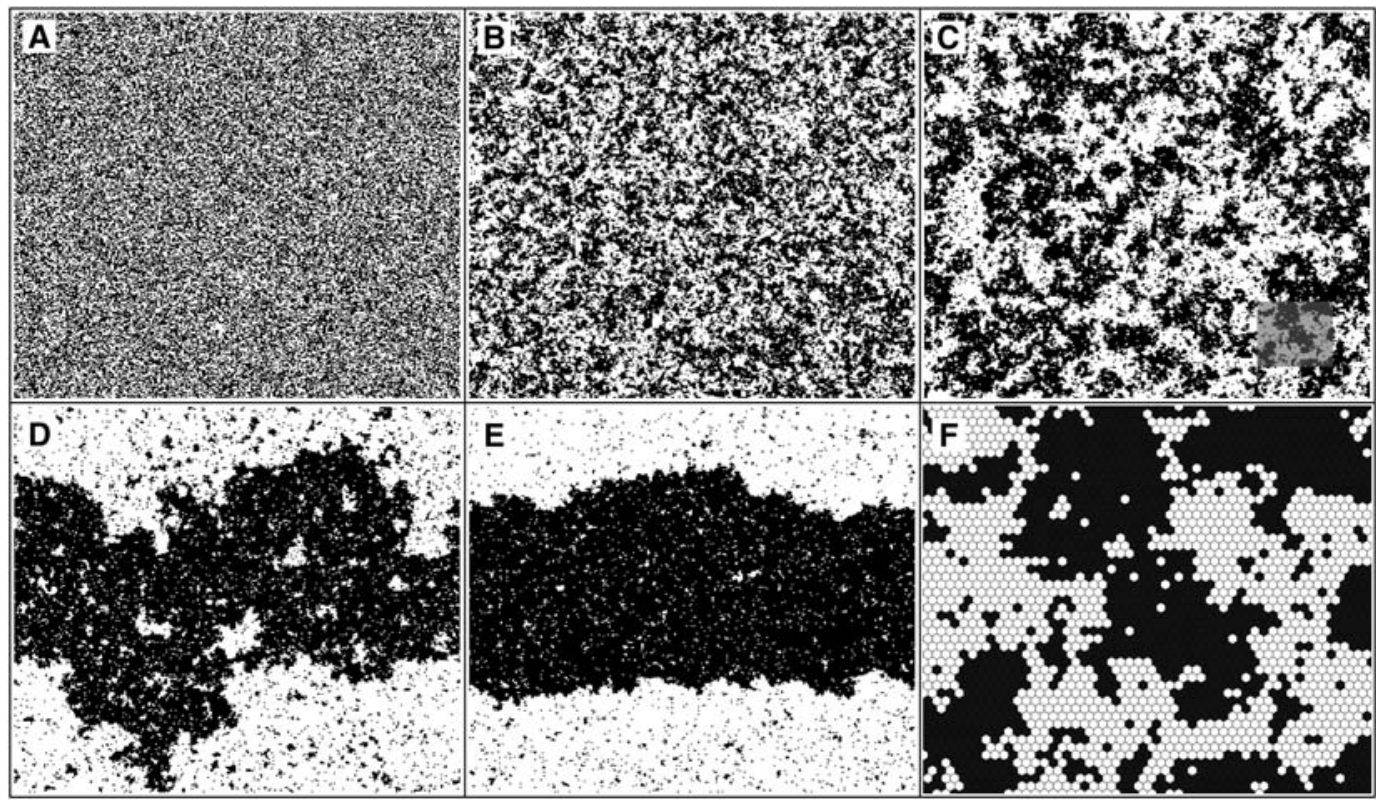

Figure 1. Lattice snapshots for Monte Carlo simulations of binary mixtures with different pairwise interaction energy $\Delta E m$. $(A)$ A random mixture $(\Delta E m=0)$ is characterized by groupings of 3 to 5 lipids. (B) An unfavorable interaction of $\Delta E m=0.4 \mathrm{kTresults}$ in clusters of 10 to 20 lipids, or a domain size of $\sim 3 \mathrm{~nm}$. (C) At $\Delta E m=$ $0.5 \mathrm{kT}$ the system is close to phase separation. An enlargement of the snapshot $(F)$ reveals large clusters composed of hundreds of lipids. $(D)$ At $\Delta E m=0.55 \mathrm{kT}$ the clusters coalesce, indicating a phase transition. (E) $\Delta E m=0.6 \mathrm{kT}$. The phase-separated mixture is characterized by a single domain of each phase. 


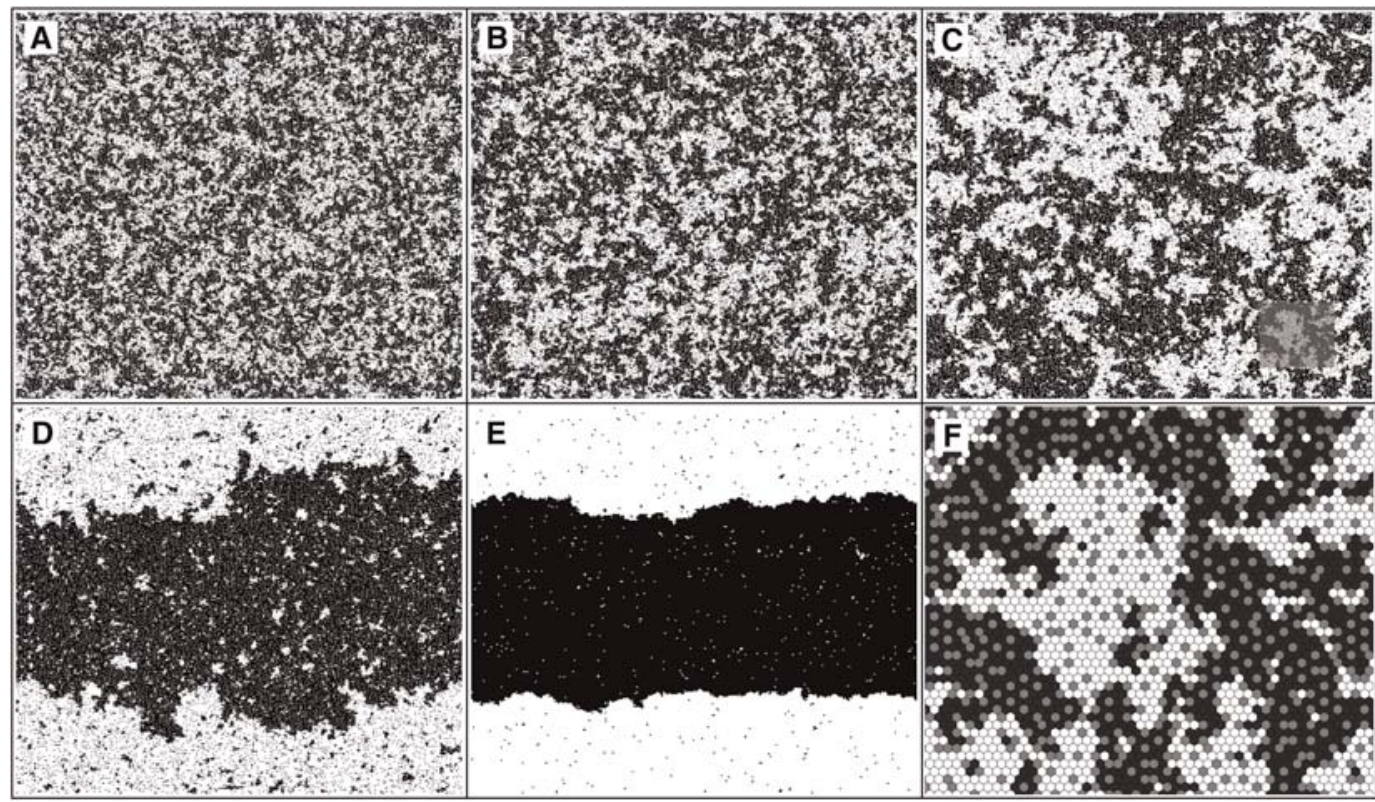

Figure 2. Lattice snapshots for Monte Carlo simulations of ternary mixtures. (E) An equimolar binary $\alpha / \beta$ mixture with an unfavorable pairwise interaction energy $\Delta E_{\alpha \beta}=0.8 \mathrm{kT}$ is characterized by coexistence of $\alpha$-rich and $\beta$-rich phases. $(A-D)$ Although maintaining the ratio of components $\alpha$ and $\beta$, a third component, $\gamma$ (which could be cholesterol), is added that interacts favorably with both $\alpha$ and $\beta\left(\Delta E_{\alpha \gamma}=-0.8 \mathrm{kT}, \Delta E_{\beta \gamma}=-1.2 \mathrm{kT}\right)$. (D) At $\chi_{\gamma}=0.20$, large clusters of $\beta$ within the $\alpha$-rich phase (and vice versa) are evident. (C) Long-range structure is broken up at $\chi_{\gamma}=0.25$. An enlargement of the snapshot $(F)$ shows clusters of hundreds of lipids, and the uneven distribution of component $\gamma$ (gray) between $\alpha$-rich (white) and $\beta$-rich (black) clusters. Further addition of component $\gamma$ reduces the size of clusters. (B) Snapshot for $\chi_{\gamma}=0.30$ and $(A) \chi_{\gamma}=0.35$.

varied. For comparison with Figure 1, Monte Carlo simulation snapshots shown in Figure 2 of a three-component mixture are arranged in an order that leads up to phase separation in Figure 2E. In these simulations, increasing cholesterol concentration leads to increased mixing of the components. At a cholesterol concentration of $35 \mathrm{~mol} \%$ the mixture shows highly nonrandom mixing, with clusters of approximately 20 lipids (Fig. 2A). Smaller cholesterol concentrations lead to increasing cluster sizes. For example, Figure 2C,F show clusters of several hundreds of lipids at $25 \mathrm{~mol} \%$ cholesterol, corresponding to a domain size of approximately $10 \mathrm{~nm}$.

Considering these two simulation studies of two- and three-component mixtures, what do we learn about the spatial distribution of lipids? First, even well-mixed or random mixtures show numerous clusters of 3 to 5 similar lipids.
Second, lipid clusters grow as the mixture is changed to approach a boundary of phase separation, but only in a limited way: Cluster sizes approach a maximal range that is similar for the mixtures with different numbers of components. However, a short distance past the phase boundary in composition space (Fig. 2) or in energy (Fig. 1) results in enormous, abrupt change in size of domains.

\section{Structured One-Phase Mixtures}

Before exploring further the nature of lipid phase separations, another possibility should be discussed: A particular spatial organization in a lipid mixture might be described as a structured one-phase region instead of phase coexistence. In this state of matter, molecules are organized on the nm and tens of nm scale, but chemical potentials can vary with mixture 
composition (unlike a two-phase tieline, in which chemical potentials are constant even as the bulk composition varies). Examples of such structured one-phase mixtures are micelles, formed as a high-order association reaction of amphiphiles (Tanford 1980), and microemulsions, which can form when surface tension is low enough compared with kT that large structure breaks up (Gelbart et al. 1999). Such structured single phases provide distinctly different possibilities for the local environment of individual molecules. As an example, DPH fluorescence increases greatly when detergent forms micelles, revealing a new environment in which DPH quantum yield is increased (London and Feigenson 1978).

An important task when modeling cell membranes is to faithfully describe the spatial organization of the system of interest. At least initially, this does not necessitate distinguishing between structured one-phase matter or twophase nanodomains of Ld + Lo. Characterizing the spatial organization would include determining the size, shape, lifetime, and connectivity of the heterogeneities, whatever the number of true phases. But the distinction between one-phase and two-phase is of interest. First, along particular directions (namely the thermodynamic tielines of a two-phase system), chemical potential of each component is constant. In this case, we can in principle measure the very informative partitioning of a variety of membrane-bound lipids and proteins between coexisting Ld and Lo phases, a potential step in establishing the rules that describe protein partitioning in cell membranes. Moreover, systematic measurements of Kp might provide an experimental test to establish whether nanoscopic domains are one-phase or two-phase. After all, in a one-phase mixture chemical potential would vary continuously without showing fixed values along particular lines. If the nanodomain region of a ternary mixture were a structured one-phase compositional region, then we would expect to find a first-order transition to a two-phase region somewhere in composition space. Sharp transitions can be biologically useful, for example, in amplification of stimuli (Stadtman and Chock 1977).
Coexisting Phases: The Special Behavior of Cholesterol in Bilayers

Phases can separate when unfavorable interactions are sufficiently large, resulting in rich phase behavior for some lipid mixtures. In a general sense, the two-chain lipids that form the bilayer phase are so very structurally different from cholesterol that we expect wide regions of immiscibility. But cholesterol, despite its high solubility in phospholipids-about 67 mol \%, or two cholesterol molecules for each phospholipid molecule at saturation for many PCs-ties up its phospholipid neighbors to accommodate what is effectively strong cholesterol-cholesterol repulsion (Huang 2002). At cholesterol concentrations greater than that of certain regular arrays, cholesterol chemical potential spikes (Ali et al. 2007). At these special concentrations, a different cholesterol-rich phase separates from the cholesterol-saturated matrix. This behavior was first described explicitly for the formation of cholesterol monohydrate from a cholesterol-saturated Lo bilayer (Huang et al. 1999), but applies to other separations of cholesterol-rich phases as well, including formation of Lo from a cholesterolsaturated $\mathrm{L} \beta$ phase.

One-, two-, and three-phase regions are found in the case of ternary mixtures with cholesterol that contain, for example, the phospholipid pairs SM + DOPC (Veatch and Keller 2005), or 1,2-dipalmitoyl-sn-glycero-3-phosphocholine (DPPC) + DOPC (Veatch and Keller 2003), or DSPC + DOPC (Zhao et al. 2007a). The interaction of DOPC with the high-melting lipid is so unfavorable that over an extensive region of composition space, phase separation of Ld and Lo occurs. Phase domains are macroscopic, and a single round domain can extend for many microns. This observation also implies line tension between Ld and Lo domains that is sufficiently large to drive their coalescence.

Figure 3 shows the phase behavior over all possible compositions at $23^{\circ} \mathrm{C}$ for the particular mixture DSPC/DOPC/cholesterol, but we note that this can be considered a general phase diagram for mixtures of cholesterol with a high- $T_{M}$ and low- $T_{M}$ lipid. As cholesterol concentration 
F.A. Heberle and G.W. Feigenson

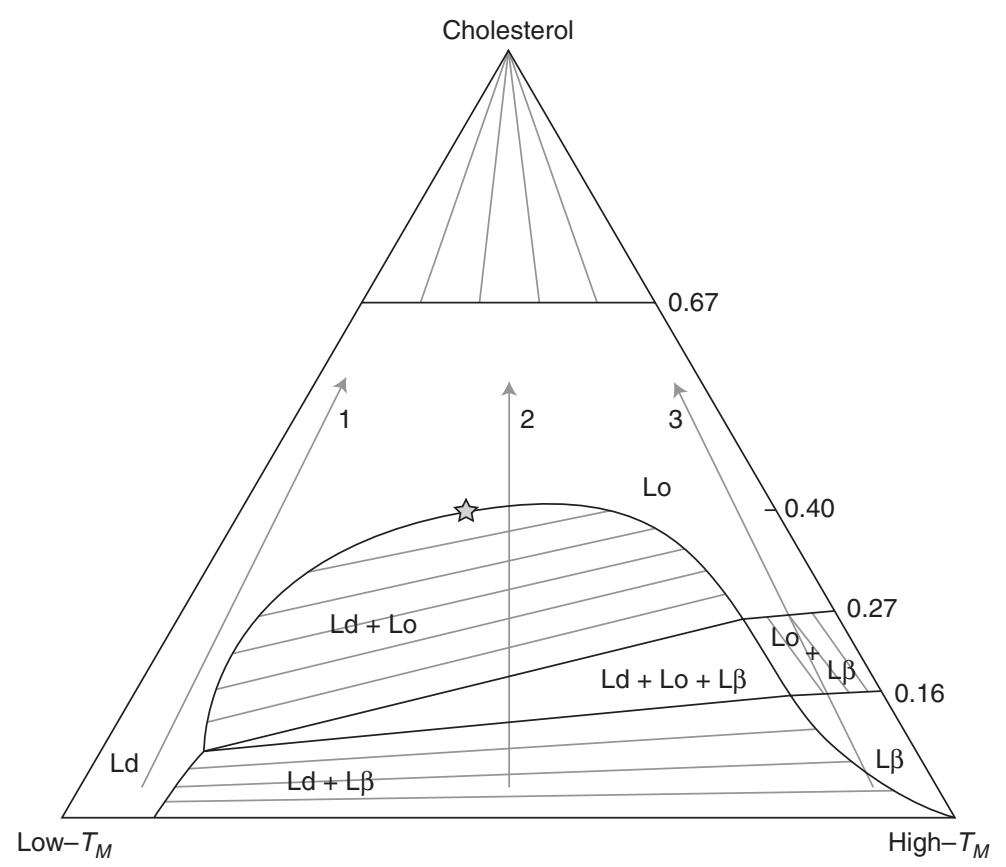

Figure 3. Illustrative phase diagram for a ternary lipid mixture containing low- and high-melting temperature lipids and cholesterol. Tielines are shown in phase-coexistence regions, and the Ld + Lo critical point is marked with a star. The effect of cholesterol addition to Ld (arrow 1), L $\beta$ (arrow 3), or phase-separated Ld + L $\beta$ mixtures (arrow 2) is discussed in the text.

increases in the Ld phase (arrow 1 in Fig. 3), apparently cholesterol continuously dissolves without phase separation right up to its maximum solubility of $67 \mathrm{~mol} \%$. The same is not true of the gel phase (arrow 3 in Fig. 3). In the $\mathrm{L} \beta$ phase, cholesterol reaches a maximum solubility of about $16 \mathrm{~mol} \%$ (for SM, DPPC, or DSPC in mixtures with DOPC, POPC, or SOPC). Cholesterol chemical potential rises steeply at this special concentration, resulting in the formation of a Lo phase having the higher cholesterol concentration of $\sim 27 \mathrm{~mol} \%$. The concentration of $16 \mathrm{~mol} \%$ corresponds to each cholesterol molecule in the gel being surrounded by about six phospholipids-every phospholipid in the gel "solvating" but one cholesterol in the cholesterol-saturated solid $\mathrm{L} \beta$ phase. Cholesterol at concentration $>16$ mol \% must be accommodated in a different type of lattice than that of the $\mathrm{L} \beta$ phase, namely that of the Lo phase. Apparently, lateral and rotational positions have larger ranges available for optimal cholesterol solvation when not dictated by the demands of the highly-ordered L $\beta$ lattice. Perhaps the details of cholesterol molecular shape make anisotropic demands on its now approximately four or fewer phospholipid neighbors in Lo, rather than the six phospholipid neighbors of the cholesterolsaturated L $\beta$ phase. Packing adjustments in Lo, unconstrained by the $\mathrm{L} \beta$ lattice, seem to enable each cholesterol to be shielded from water by fewer lipid headgroups.

As cholesterol concentration increases, eventually reaching its maximum solubility in the $\mathrm{L} \beta$ phase, the cholesterol also responds to the presence of any coexisting Ld phase. For example, Figure 3 arrow 2 corresponds to the addition of cholesterol to a two-phase mixture of Ld (DSPC-saturated DOPC) and L $\beta$ (DOPC-saturated DSPC). In this interesting situation, the cholesterol has modest preference for $\mathrm{L} \beta$ over $\mathrm{Ld}$, but dissolves into both phases. In $\mathrm{Ld}$ and $\mathrm{L} \beta$, cholesterol chemical potential 
does not rise very steeply as its concentration increases (until near saturation in $\mathrm{L} \beta$ ), and in fact is lower in $\mathrm{L} \beta$ than in $\mathrm{Ld}$, as evidenced by its higher concentration in the $\mathrm{L} \beta$ phase that is in equilibrium with Ld phase. However, a steep jump in cholesterol chemical potential occurs in the $\mathrm{L} \beta$ phase for concentration greater than $\sim 0.16 \mathrm{~mol} \%$. Once the Lo phase separates out from $\mathrm{L} \beta$, more added cholesterol enters both Ld and Lo, with an initial preference of about 2.5 for Lo over Ld, a preference that gets smaller as cholesterol concentration increases in the Ld phase. In the DOPC-rich Ld phase that is in equilibrium with a DSPC-rich or SM-rich Lo phase, at a cholesterol concentration of about $40 \mathrm{~mol} \%$, the phases become indistinguishable; this is the critical point. In the POPC-rich or SOPC-rich Ld phase that is in equilibrium with a DSPC-rich Lo phase, the lower cholesterol concentration of about $30 \mathrm{~mol} \%$ makes the Ld and Lo phases indistinguishable (Heberle et al. 2010).

However, in the POPC- or SOPC-containing mixtures, in the $\mathrm{Ld}+$ Lo region far from this critical point, another interesting behavior is manifest: the phase domains are smaller than the wavelength of light, about 200$300 \mathrm{~nm}$, and so are not resolved in ordinaryresolution optical microscopy. However, FRET (Silvius 2003), fluorescence anisotropy (de Almeida et al. 2003), ESR spin label order parameter and rotational diffusion (Heberle et al. 2010), and $\delta$-Lysin-induced dye efflux (Pokorny et al. 2006) are all consistent with coexistence of Ld and Lo phases.

If nanodomains of Ld + Lo can be treated as two-phase coexistence, some questions arise:

1. Given that minimization of domain perimeter is driven by lowering the free energy from line tension between Ld and Lo domains, what is an opposing interaction that would become unfavorable as domain size grows?

The result of such competing interactions would be modulated phase behavior. Depending on the relative magnitude of the competing terms, a variety of domain morphologies and size scales can be observed, including round domains, straight lines, branched lines, and maze patterns (Seul and Andelman 1995). Possible candidates for such an interaction that becomes unfavorable as domain size grows can be identified: (1) Lipid intrinsic curvature has been proposed to favor domains of a particular size (Brewster and Safran 2010); (2) The special demand of each individual cholesterol molecule to be "solvated" by neighboring lipids is not symmetrical around each cholesterol molecule, and each "lipid solvation shell" could interact unfavorably with others to oppose domain growth.

2. To what extent are the chemical and physical properties of any nanodomains in POPCand SOPC-containing mixtures similar to, or different from, the macroscopic phase domains of $\mathrm{Ld}+\mathrm{Lo}$ in the related mixtures $\mathrm{SM} / \mathrm{DOPC} / \mathrm{chol}$ or DSPC/DOPC/chol, for example?

Simons and colleagues have emphasized that the partition of "raft-associated" proteins in cell membranes differs from the partition observed in simple lipid mixture models of cell membranes (Kaiser et al. 2009). But perhaps protein partition behavior for coexisting macroscopic domains is misleading. This hypothesis can be tested by comparing the partitioning of even simple fluorescent dyes in the nanoscopic and macroscopic mixtures. Of great interest would be to find the Kp of various lipids, peptides, and proteins. Furthermore, the physical properties of order parameter and diffusion coefficient differences between nanoscopic and macroscopic domains might inform as to the fundamental domain characteristics. For example, the order parameter $S_{0}$ of the acyl chain spin label 16-PC responds in a similar way to the phase separation of either macroscopic or nanoscopic domains, yet the nanoscopic Ld-phase domains show greater order than macroscopic Ld-phase domains (Fig. 4).

3. Are interesting morphologies observed when Ld and Lo phases coexist?

Given two-phase coexistence, Seul and Andelman (1995) and others have described 
F.A. Heberle and G.W. Feigenson

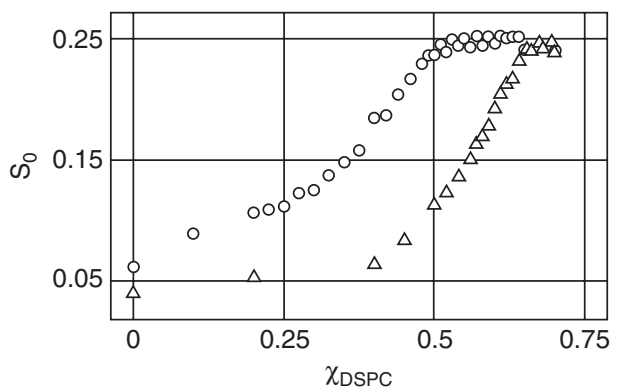

Figure 4. ESR reveals similarity of macroscopic and nanoscopic phase properties. Compositional trajectories run in the approximate direction of Ld + Lo tielines (see Fig. 3) and differ only in the identity of the low- $T_{M}$ lipid. Composition-dependent order parameters obtained from ESR spectral simulations in DSPC/DOPC/chol (triangles) and DSPC/POPC/ chol (circles).

modulation of spatial organization that shows a number of possible morphologies, including the possibility of progression through different morphologies. For mixtures that show a range of size scales, for example the four-component mixture DSPC/ DOPC/POPC/chol, the extremes of fluid phase morphology are known: micronsized, round domains at $0 \mathrm{~mol} \% \mathrm{POPC}$, and domains much smaller than $300 \mathrm{~nm}$ (and likely in the range of $2-5 \mathrm{~nm}$ ) at $0 \mathrm{~mol} \%$ DOPC. However, in the intermediate regime, different morphologies are frequently observed (Fig. 5). The systematic examination of such behavior in lipid-only models might inform a search for similar morphologies in cell membranes.

4. Are the domains observed with light microscopy strongly sensitive to intense illumination?

For any imaging techniques in which the question is a transition from "close-tophase-separated" to actually phase-separated, we expect sensitivity to light-induced macroscopic domains. This artifact can be minimized by use of low dye concentration, multiphoton excitation, and sensitive cameras (Morales-Penningston et al. 2010).

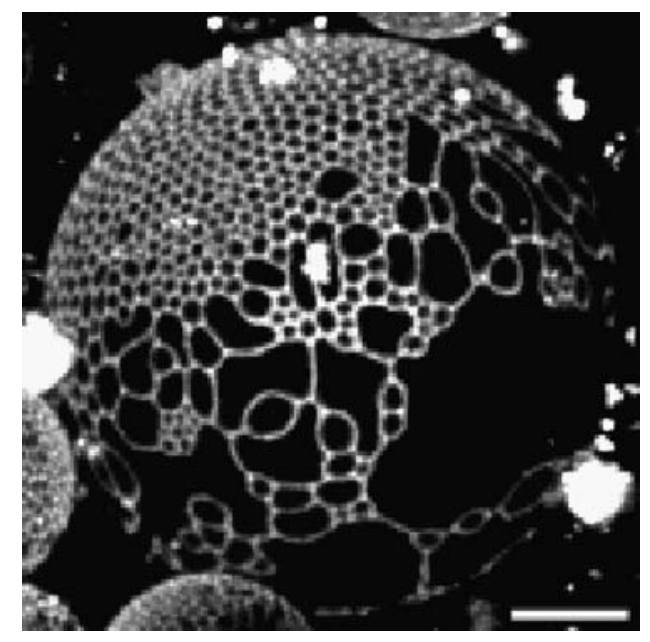

Figure 5. Confocal microscopy of giant unilamellar vesicles reveals interesting domain morphology in a four-component mixture that is intermediate in size between the smallest nanoscopic domains of DSPC/POPC/chol and the macroscopic domains of DSPC/DOPC/chol. Vesicle composition DSPC/ DOPC $/$ POPC $/$ chol $=45 / 4.5 / 25.5 / 25$. Scale bar 10 microns.

Value of a Phase Diagram for a Four-Component Bilayer Mixture

Because DSPC/DOPC/chol has macroscopic Ld + Lo phase separation, whereas DSPC/ POPC/chol has nanometer-scale Ld + Lo separation, a minimal system with domain size controlled by composition would be the fourcomponent mixtures DSPC/DOPC/POPC/ chol. Finding the boundaries of phase regions in four-component mixtures will be dataintensive because a three-dimensional composition space must be examined. In addition to phase boundaries, domain size scales must be determined; GUV imaging and FRET are useful for obtaining such information. Domain lifetime distributions are necessary for understanding their role in the enzymatic activity of membrane proteins. Are there distinct protein behaviors in response to distinct lipid phase behaviors, or does a short domain lifetime effectively present the protein with an averaged environment? 


\section{CONCLUSIONS}

It is clear that cell membranes possess domains with a range of sizes. Compositional heterogeneities also occur in lipid-only bilayer mixtures, with a range of sizes and perhaps other properties. Model mixtures in which domain size is controlled by composition from nanometerscale to microns would provide a valuable experimental system for examining this aspect of membrane rafts. So far, no such systematic study has been published, though we have recently identified a four-component mixture that meets this criterion. Also of interest for the behavior of cell membranes, even single phase nonideal lipid mixtures can be induced to increase the size of their heterogeneities. Here, a case that plagues the use of fluorescence microscopy is the introduction of artifactual visible domains by intense illumination (Zhao et al. 2007b). This might be caused by free radical initiated polymerization that starts from the reactive excited singlet state (Ayuyan and Cohen 2006). More interesting examples come from aggregating ganglioside by binding cholera toxin B subunit, resulting in large domains (Hammond et al. 2005). Such controlled increase of domain size by protein binding or cross-linking could be used by cells in a controlled fashion to effect cellular functions (Lingwood et al. 2008). Furthermore, perhaps the cell has mechanisms for the complementary role of controlled decrease of domain size, for example, via protein "obstacles" that interfere with phase separation.

\section{ACKNOWLEDGMENTS}

We gratefully acknowledge the help of J. Huang in teaching us details of the Monte Carlo simulation, and J. Wu for the GUV image. Support was received from research awards from the National Institutes of Health R01 GM077198 and the National Science Foundation MCB 0842839 (G.W.F.). F.A.H. was supported in part by National Institutes of Health research award 1-T32-GM08267.

\section{REFERENCES}

Abney JR, Scalettar BA. 1995. Fluctuations and membrane heterogeneity. Biophys Chem 57: 27-36.

Ali MR, Cheng KH, Huang J. 2007. Assess the nature of cholesterol-lipid interactions through the chemical potential of cholesterol in phosphatidylcholine bilayers. Proc Natl Acad Sci 104: 5372-5377.

Ayuyan AG, Cohen FS. 2006. Lipid peroxides promote large rafts: Effects of excitation of probes in fluorescence microscopy and electrochemical reactions during vesicle formation. Biophys J 91: 2172-2183.

Brewster R, Safran SA. 2010. Line active hybrid lipids determine domain size in phase separation of saturated and unsaturated lipids. Biophys J 98: L21-L23.

Collins MD, Keller SL. 2008. Tuning lipid mixtures to induce or suppress domain formation across leaflets of unsupported asymmetric bilayers. Proc Natl Acad Sci 105: 124- 128 .

de Almeida RF, Fedorov A, Prieto M. 2003. Sphingomyelin/ phosphatidylcholine/cholesterol phase diagram: Boundaries and composition of lipid rafts. Biophys $J$ 85: 2406-2416.

Elson EL, Fried E, Dolbow JE, Genin GM. 2010. Phase separation in biological membranes: integration of theory and experiment. Annu Rev Biophys 39: 207-226.

Fan J. Sammalkorpi M. 2010. Influence of nonequilibrium lipid transport, membrane compartmentalization, and membrane proteins on the lateral organization of the plasma membrane. Physical Review E 81: 011908.

Feigenson GW, Buboltz JT. 2001. Ternary phase diagram of dipalmitoyl-PC/dilauroyl-PC/cholesterol: Nanoscopic domain formation driven by cholesterol. Biophys $J \mathbf{8 0}$ 2775-2788.

Frolov VA, Chizmadzhev YA, Cohen FS, Zimmerberg J. 2006. "Entropic traps" in the kinetics of phase separation in multicomponent membranes stabilize nanodomains. Biophys J 91: 189-205.

Gelbart WM, Sear RP, Heath JR, Chaney S. 1999. Array formation in nano-colloids: Theory and experiment in 2D. Faraday Discuss Chem Soc 112: 299-307.

Gennis RB. 1989. Biomembranes: Molecular structure and function. Springer-Verlag, New York.

Guggenheim EA. 1952. Mixtures; the theory of the equilibrium properties of some simple classes of mixtures, solutions and alloys. Clarendon, Oxford, United Kingdom.

Haluska CK, Schroder AP, Didier P, Heissler D, Duportail G, Mely Y, Marques CM. 2008. Combining fluorescence lifetime and polarization microscopy to discriminate phase separated domains in giant unilamellar vesicles. Biophys J 95: $5737-5747$.

Hammond AT, Heberle FA, Baumgart T, Holowka D, Baird B, Feigenson GW. 2005. Crosslinking a lipid raft component triggers liquid ordered-liquid disordered phase separation in model plasma membranes. Proc Natl Acad Sci 102: 6320-6325.

Heberle FA, Wu J, Goh SL, Petruzielo RS, Feigenson GW. 2010. Comparison of three ternary lipid bilayer mixtures: FRET and ESR reveal nanodomains. Biophys J doi:101016/ jbpj201009064. 
F.A. Heberle and G.W. Feigenson

Honerkamp-Smith AR, Cicuta P, Collins MD, Veatch SL, den Nijs M, Schick M, Keller SL. 2008. Line tensions, correlation lengths, and critical exponents in lipid membranes near critical points. Biophys J 95: 236-246.

Huang J. 2002. Exploration of molecular interactions in cholesterol superlattices: Effect of multibody interactions. Biophys J 83: 1014-1025.

Huang J, Feigenson GW. 1993. Monte Carlo simulation of lipid mixtures: finding phase separation. Biophys J 65: $1788-1794$.

Huang C, Li S. 1999. Calorimetric and molecular mechanics studies of the thermotropic phase behavior of membrane phospholipids. Biochim Biophys Acta 1422: 273-307.

Huang J, Buboltz JT, Feigenson GW. 1999. Maximum solubility of cholesterol in phosphatidylcholine and phosphatidylethanolamine bilayers. Biochim Biophys Acta 1417: 89-100.

Imperio A, Reatto L. 2006. Microphase separation in twodimensional systems with competing interactions. $J$ Chem Phys 124: 164712.

Kaiser HJ, Lingwood D, Levental I, Sampaio JL, Kalvodova L, Rajendran L, Simons K. 2009. Order of lipid phases in model and plasma membranes. Proc Natl Acad Sci 106: $16645-16650$.

Kiessling V, Wan C, Tamm LK. 2009. Domain coupling in asymmetric lipid bilayers. Biochim Biophys Acta 1788: $64-71$.

Kinosita K Jr, Kawato S, Ikegami A. 1984. Dynamic structure of biological and model membranes: Analysis by optical anisotropy decay measurement. Adv Biophys 17: 147203.

Lingwood D, Simons K. 2010. Lipid rafts as a membraneorganizing principle. Science 327: 46-50.

Lingwood D, Ries J, Schwille P, Simons K. 2008. Plasma membranes are poised for activation of raft phase coalescence at physiological temperature. Proc Natl Acad Sci 105: 10005-10010.

London E, Feigenson GW. 1978. Fluorescence quenching of $\mathrm{Ca}^{2+}$-ATPase in bilayer vesicles by a spin-labeled phospholipid. FEBS Lett 96: 51-54.

London E, Feigenson GW. 1981. Fluorescence quenching in model membranes. 1. Characterization of quenching caused by a spin-labeled phospholipid. Biochemistry 20: 1932-1938.

Manz BN, Groves JT. 2010. Spatial organization and signal transduction at intercellular junctions. Nat Rev Mol Cell Biol 11: 342-352.

Marsh D. 2009. Cholesterol-induced fluid membrane domains: A compendium of lipid-raft ternary phase diagrams. Biochim Biophys Acta 1788: 2114-2123.

McLaughlin S, Murray D. 2005. Plasma membrane phosphoinositide organization by protein electrostatics. Nature 438: 605-611.

Morales-Penningston NF, Wu J, Farkas ER, Goh SL, Konyakhina TM, Zheng JY, Webb WW, Feigenson GW. 2010. GUV preparation and imaging: Minimizing artifacts. Biochim Biophys Acta 1798: 1324-1332.
Phillips MC, Ladbrooke BD, Chapman D. 1970. Molecular interactions in mixed lecithin systems. Biochim Biophys Acta 196: 35-44.

Pokorny A, Yandek LE, Elegbede AI, Hinderliter A, Almeida PF. 2006. Temperature and composition dependence of the interaction of delta-lysin with ternary mixtures of sphingomyelin/cholesterol/POPC. Biophys J 91: 21842197.

Polozova A, Litman BJ. 2000. Cholesterol dependent recruitment of di22:6-PC by a $\mathrm{G}$ protein-coupled receptor into lateral domains. Biophys J 79: 2632-2643.

Sahl SJ, Leutenegger M, Hilbert M, Hell SW, Eggeling C. 2010. Fast molecular tracking maps nanoscale dynamics of plasma membrane lipids. Proc Natl Acad Sci 107: 6829-6834.

Schneider DJ, Freed JH. 1989. Calculating slow motional magnetic resonance spectra. A user's guide. In Spin labeling: Theory and application (ed Reuben J). Plenum, New York.

Semrau S, Idema T, Schmidt T, Storm C. 2009. Membranemediated interactions measured using membrane domains. Biophys J 96: 4906-4915.

Seul M, Andelman D. 1995. Domain shapes and patterns: The phenomenology of modulated phases. Science 267: $476-483$.

Sheetz MP. 1993. Glycoprotein motility and dynamic domains in fluid plasma membranes. Annu Rev Biophys Biomol Struct 22: 417-431.

Silvius JR. 2003. Fluorescence energy transfer reveals microdomain formation at physiological temperatures in lipid mixtures modeling the outer leaflet of the plasma membrane. Biophys J 85: 1034-1045.

Simons K, van Meer G. 1988. Lipid sorting in epithelial cells. Biochemistry 27: 6197-6202.

Stadtman ER, Chock PB. 1977. Superiority of interconvertible enzyme cascades in metabolic regulation: analysis of monocyclic systems. Proc Natl Acad Sci 74: 2761-2765.

Tanford C. 1980. The hydrophobic effect: The formation of micelles and biological membranes, 2nd ed. Wiley, New York.

Tenchov BG, Koynova RD. 1985. The effect of nonideal lateral mixing on the transmembrane lipid asymmetry. Biochim Biophys Acta 815: 380-391.

van Meer G, Voelker DR, Feigenson GW. 2008. Membrane lipids: Where they are and how they behave. Nat Rev Mol Cell Biol 9: 112-124.

Veatch SL, Keller SL. 2003. Separation of liquid phases in giant vesicles of ternary mixtures of phospholipids and cholesterol. Biophys J 85: 3074-3083.

Veatch SL, Keller SL. 2005. Miscibility phase diagrams of giant vesicles containing sphingomyelin. Phys Rev Lett 94: 148101.

Veatch SL, Gawrisch K, Keller SL. 2006. Closed-loop miscibility gap and quantitative tie-lines in ternary membranes containing diphytanoyl PC. Biophys $J$ 90: $4428-4436$.

Vist MR, Davis JH. 1990. Phase equilibria of cholesterol/ dipalmitoylphosphatidylcholine mixtures: $2 \mathrm{H}$ nuclear 
Phase Separation in Lipid Membranes

magnetic resonance and differential scanning calorimetry. Biochemistry 29: 451-464.

Wenk MR. 2005. The emerging field of lipidomics. Nat Rev Drug Discov 4: 594-610.

Wu SH, McConnell HM. 1975. Phase separations in phospholipid membranes. Biochemistry 14: 847-854.

Zhao J, Wu J, Heberle FA, Mills TT, Klawitter P, Huang G, Costanza G, Feigenson GW. 2007a. Phase studies of model biomembranes: Complex behavior of DSPC/ DOPC/cholesterol. Biochim Biophys Acta 1768: 2764-2776.

Zhao J, Wu J, Shao H, Kong F, Jain N, Hunt G, Feigenson G. 2007b. Phase studies of model biomembranes: Macroscopic coexistence of Lalpha + Lbeta, with light-induced coexistence of Lalpha + Lo Phases. Biochim Biophys Acta 1768: $2777-2786$. 


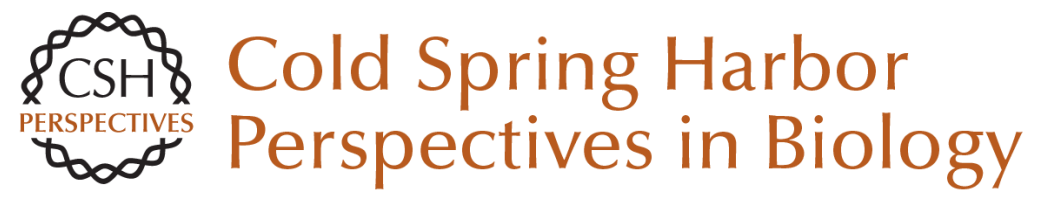

\section{Phase Separation in Lipid Membranes}

Frederick A. Heberle and Gerald W. Feigenson

Cold Spring Harb Perspect Biol 2011; doi: 10.1101/cshperspect.a004630 originally published online March 2, 2011

\section{Subject Collection The Biology of Lipids}

Role of Lipids in Virus Replication Maier Lorizate and Hans-Georg Kräusslich

Model Answers to Lipid Membrane Questions Ole G. Mouritsen

\section{Glycosphingolipid Functions} Clifford A. Lingwood

Regulation of Cholesterol and Fatty Acid Synthesis

Jin Ye and Russell A. DeBose-Boyd

\section{Lipid-Mediated Endocytosis}

Helge Ewers and Ari Helenius

\section{Fluorescence Techniques to Study Lipid \\ Dynamics}

Erdinc Sezgin and Petra Schwille

Lysosomal Lipid Storage Diseases

Heike Schulze and Konrad Sandhoff

\section{Distribution and Functions of Sterols and} Sphingolipids

J. Thomas Hannich, Kyohei Umebayashi and Howard Riezman
Membrane Organization and Lipid Rafts Kai Simons and Julio L. Sampaio

Shotgun Lipidomics on High Resolution Mass

Spectrometers

Dominik Schwudke, Kai Schuhmann, Ronny

Herzog, et al.

Glycosphingolipid Functions Clifford A. Lingwood

Phosphoinositides in Cell Architecture Annette Shewan, Dennis J. Eastburn and Keith Mostov

Synthesis and Biosynthetic Trafficking of Membrane Lipids Tomas Blom, Pentti Somerharju and Elina Ikonen

Lipid Polymorphisms and Membrane Shape Vadim A. Frolov, Anna V. Shnyrova and Joshua Zimmerberg

Specificity of Intramembrane Protein-Lipid Interactions

Francesc-Xabier Contreras, Andreas Max Ernst, Felix Wieland, et al.

Dynamic Transbilayer Lipid Asymmetry Gerrit van Meer

For additional articles in this collection, see http://cshperspectives.cshlp.org/cgi/collection/

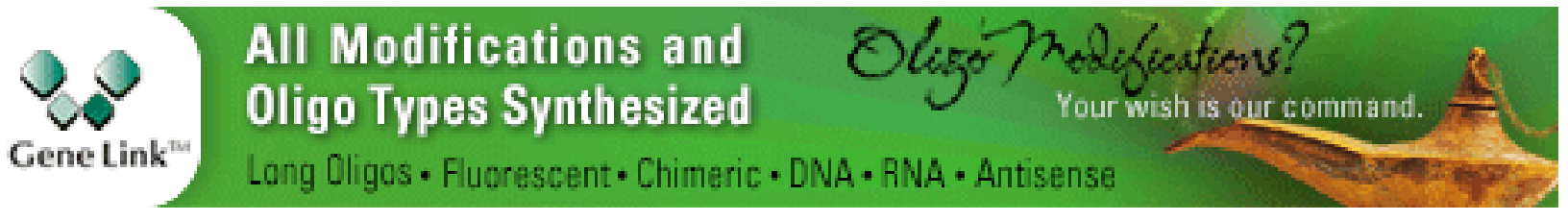

Copyright @ 2011 Cold Spring Harbor Laboratory Press; all rights reserved 